\title{
Periodontal status of children with primary immunodeficiencies: a systematic review
}

\author{
H. Halai ${ }^{1} \cdot$ C. Somani ${ }^{2} \cdot$ N. Donos ${ }^{1} \cdot$ Luigi Nibali $^{1,3}$ C \\ Received: 3 June 2019 / Accepted: 5 August 2019/Published online: 18 October 2019 \\ (C) The Author(s) 2019
}

\begin{abstract}
Objective The aim of this systematic review was to appraise the existing literature on periodontal disease in children affected by different types of neutrophil-associated primary immunodeficiencies (PIDs).

Methods A PRESS-validated search strategy was developed to search through databases MEDLINE, EMBASE, Cochrane Central Register of Controlled Trials, LILACS, Google Scholar and Open Grey. All included studies were assessed for methodological quality and risk of bias.

Results One hundred eighteen articles reporting on 160 PID patients were included for qualitative analysis. The majority (70\%) were individual case reports. Clinical and radiographic manifestations of the periodontal disease included poor oral hygiene, generalised alveolar bone loss, severe gingival inflammation, increased pocket depths, tooth mobility and gingival recession. For most studies, the primary intervention was periodontal treatment in the form of scaling and root planing or dental extractions. Stabilisation of the periodontal condition varied between different PIDs. In severe congenital neutropenia (SCN), $61 \%$ of cases reported stabilisation of the periodontal condition, while for all other PIDs, 'stability' was reported in less than $43 \%$ of cases. Conclusion The published literature suggests that patients with PIDs can present with severe periodontitis and that conventional treatment approaches have limited benefits.
\end{abstract}

Keywords Immune defects $\cdot$ Inflammatory diseases $\cdot$ Paediatrics $\cdot$ Periodontium $\cdot$ Therapies

\section{Introduction}

Primary immunodeficiency diseases (PIDs) are a group of rare disorders encompassing inherited and congenital forms of neutropenia. In PIDs, there is an intrinsic defect of the immune

Electronic supplementary material The online version of this article (https://doi.org/10.1007/s00784-019-03055-z) contains supplementary material, which is available to authorized users.

Luigi Nibali

luigi.nibali@kcl.ac.uk

1 Centre for Immunobiology and Regenerative Medicine,Centre for Oral Clinical Research, Institute of Dentistry, Queen Mary University London (QMUL), London, UK

2 Department of Paediatric Dentistry, Royal London Dental Hospital, London, UK

3 Centre for Oral Immunology \& Regenerative Medicine, Clinical Oral Research Centre, Institute of Dentistry, Queen Mary University of London (QMUL), London, UK system, such as failure or absence of the cellular defences against infection. PIDs have been associated with more than 200 gene defects and manifest in over 180 different phenotypes [1]. The rarer and more severe forms of PIDs present in childhood and are characterised by increased susceptibility to infection and autoimmune disease [1]. Dental and oral manifestations of PIDs occur frequently and include oral ulceration, developmental abnormalities (such as delayed formation of teeth, enamel hypoplasia and hypocalcification) [2-4] and periodontal disease [5].

As neutrophils play a key role in the innate immune response against periodontal pathogens, children with neutropenia and neutrophil dysfunction-associated PIDs (summarised in Table 1) are thought to be the most susceptible to periodontal disease [6].

The current literature suggests that these patients' response to conventional periodontal therapy is suboptimal and unpredictable [7-9], leading to early tooth loss and a subsequent debilitating effect on mastication, aesthetics and quality of life. However, most of the published papers on periodontitis in children with neutrophil-associated immunodeficiencies are 


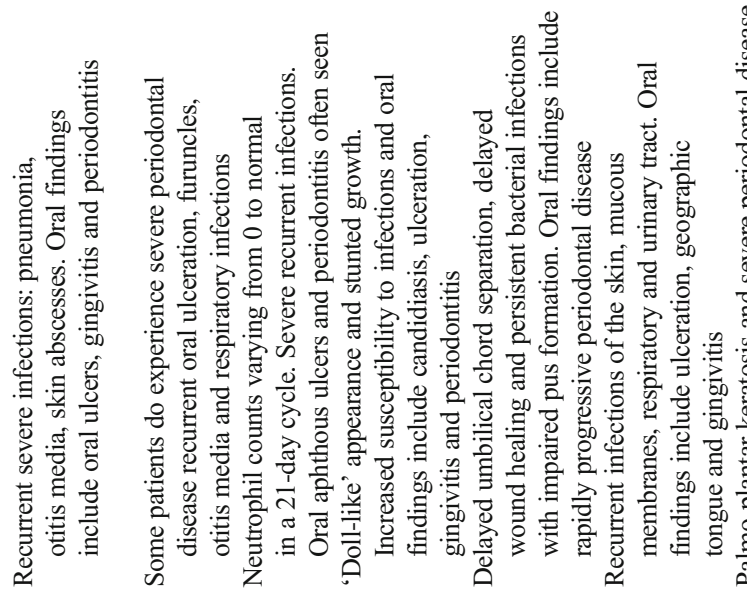

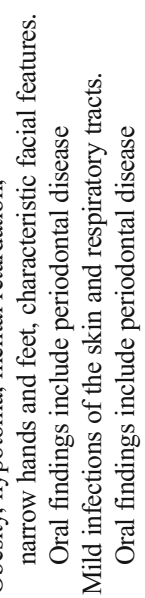

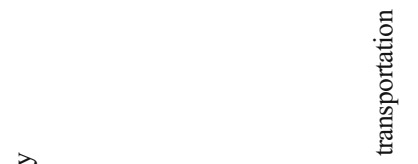

兘

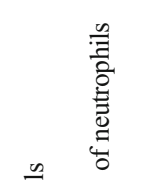

产<smiles>C1CCCCC1</smiles>

总 带

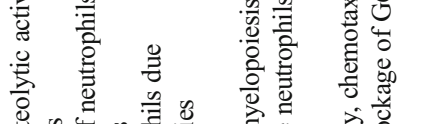

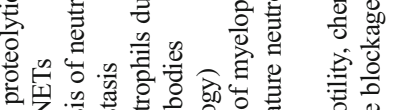

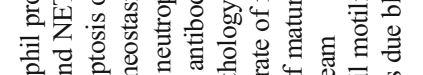

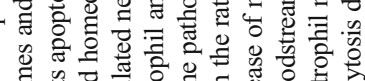

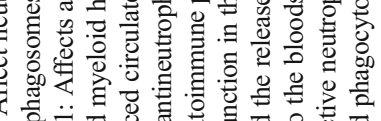

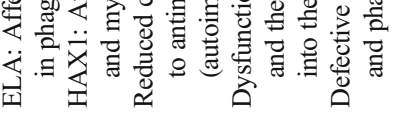

竞署

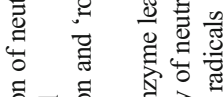

兽零

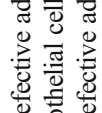

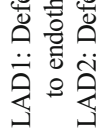

要离

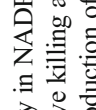

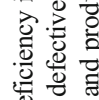

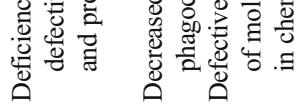

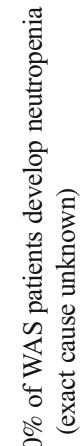

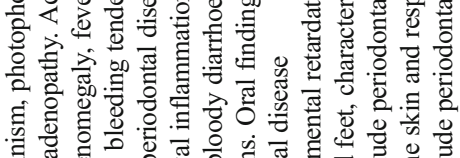

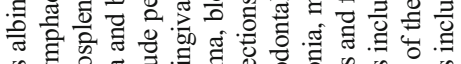

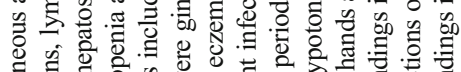

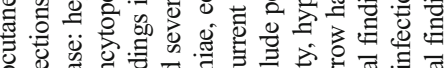

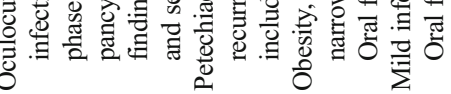

莺

当

賞

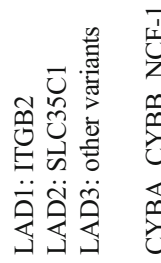

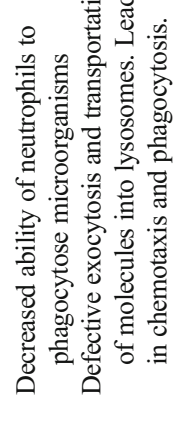

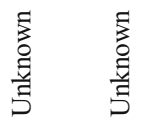

站

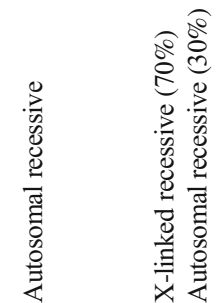

品

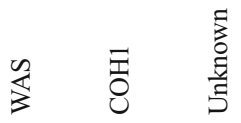

Z्.

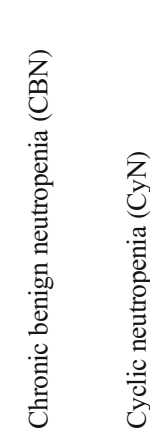

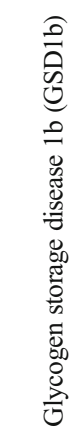

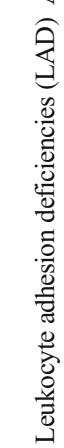

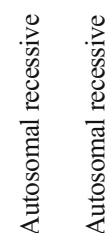

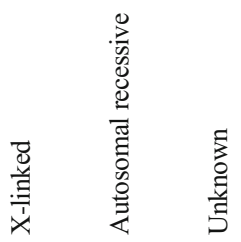

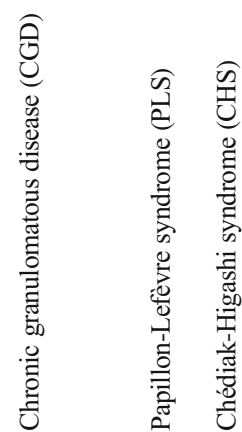

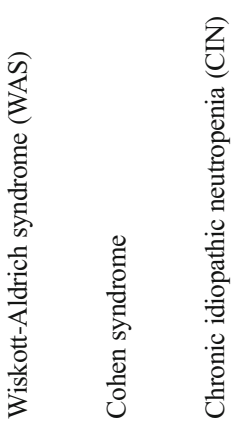


limited to case reports and case series. These have not been extensively and systematically appraised. Consequently, there is a lack of comprehensive data on the prevalence of periodontal disease in patients with such PIDs and on response to treatment.

The aim of this systematic review was to provide a comprehensive assessment of the characteristics and management of periodontitis in neutrophil-associated PID patients. In turn, this may help in early diagnosis and treatment of affected children and identify areas in need of further research.

\section{Materials and methods}

The PRISMA checklist [10] was followed in conducting this systematic review. The review protocol was registered with PROSPERO (ref no. CRD42016041252).

\section{Review question}

What is the prevalence and features of periodontitis and the response to periodontal treatment in children with neutrophilassociated PIDs?

\section{Eligibility criteria}

Studies were selected according to the defined criteria below:

- Study designs

Randomised controlled trials (RCTs) and controlled clinical trials (CCTs), case-control, cross-sectional, case reports and case series included.

\section{- Participants}

Studies examining and reporting human children participants (age $\leq 16$ years old) with a neutrophil-associated PID (as per Table 1) were included.

- Interventions

Interventions of interest involved treatment of periodontal disease taking a broad perspective (for example, oral hygiene instruction or root surface debridement). Other types of interventions were considered on a case-by-case basis (for example full mouth extractions or surgery) to what existed in the literature.

- Comparators
If any experimental studies existed in the literature, the comparator of interest would be no periodontal treatment provided.

\section{- Outcomes}

Studies were only included if at least one clinical or radiographic feature of periodontal disease was reported (e.g. gingival inflammation, increased pocket depths, alveolar bone loss).

- Report characteristics

No restriction on publication date, publication status, setting, language or geographical location was applied.

\section{Information sources}

The initial search was conducted through databases: MEDLINE, EMBASE, Cochrane Central Register of Controlled Trials, LILACS, Google Scholar and Open Grey up until 28 February 2018. To ensure literature saturation, the electronic search was complemented by a search through reference lists of included studies and previous reviews within the topic.

\section{Search strategy}

The developed search strategy (supporting material 2) was peer-reviewed by a specialist librarian, using the PeerReview of Electronic Search Strategies (PRESS) standard [11].

\section{Study selection process}

Step 1: Initial screening of potentially suitable titles and abstracts. A kappa score was calculated to assess the agreement between the two reviewers (authors $\mathrm{HH}$ and CS)

Step 2: Full paper screening, verification of eligibility and data extraction of those studies identified in step 1. Ten randomly selected studies (using an online random number generator-www.random.org) were used to calibrate the two reviewers. A kappa score was again calculated. One reviewer, $\mathrm{HH}$, completed full paper screening of the remaining studies.

In case of a disagreement between reviewers, a third reviewer (author LN) judged study inclusion.

A standardised data extraction form was used to record study characteristics, design, patients included, clinical and radiographic diagnoses, treatment provided, response to treatment and patient-reported outcomes. 
The reviewers were not blinded to the journal titles, authors or institutions. Overlapping or duplicate reports were identified in the extraction process and data was only included once. Authors were contacted if clarification was required; any missing information was noted as 'NR- not reported'.

\section{Outcomes}

The primary outcomes assessed were as follows:

- Clinical and radiographic features of periodontal disease in children with PIDs

- Periodontal treatment response (clinical and patientreported outcomes) in children with PIDs and periodontal disease

\section{Periodontal phenotype definition}

Owing to heterogeneous reporting of periodontal status across papers including definition based on probing pocket depths (PPD) and clinical attachment loss (CAL), or narrative descriptions such as 'periodontal disease was present' or 'deep pockets', the definitions below were used to summarise studies:

- Gingival inflammation: defined as 'gingivitis, 'gingival redness', 'gingival oedema', 'gingival swelling', 'gingival erythema', 'bleeding on probing' and any other term denoting inflammation of the gingival tissues.

- Periodontitis: defined as PPDs $>3 \mathrm{~mm}$ or CAL $>1 \mathrm{~mm}$ in at least one site (18), 'periodontitis or periodontal disease was present', 'increased pocket or probing depths', 'attachment loss', radiographic evidence of bone loss or 'bone destruction' and any other terms denoting increased pocket depths, clinical attachment loss or alveolar bone loss.

- Oral hygiene status: 'Poor or suboptimal' defined as plaque indices $>2$ (19), full mouth plaque scores (FMPS) $>30 \%$ (20), generalised plaque and or calculus. 'Fair' defined as plaque indices of $1-2$, FMPS $\leq 30 \%$ and $>20 \%$, localised plaque and or calculus. 'Good' defined as plaque index $<1$, FMPS $\leq 20 \%$, minimal plaque and or calculus.

- Response to treatment: 'deterioration' defined as persistence of gingival inflammation, non-resolution of pocket depths, further clinical attachment loss and further tooth loss; 'stabilisation' defined as absence of gingival inflammation, resolution of pocket depths, no further clinical attachment loss and no further tooth loss.

All other terms to describe severity of gingival inflammation and periodontitis were assessed on a case-by-case basis.

\section{Data synthesis}

Narrative synthesis with text and tables were used to explore the association and findings within and between included studies and the results were stratified for each PID. In this article, the results have been summarised for each measured outcome. No meta-analysis was performed as a lack of homogeneity existed in reporting between studies.

\section{Risk of bias and quality assessment}

The qualities of the included studies were assessed using specific criteria for each study design as listed in supporting material 3. Each study was given a final score indicating the relative research quality. Publication bias and outcome reporting bias also affected the strength of the results, the authors accept this presents a limitation of the systematic review, and wherever possible attempted to detect and mitigate these biases.

\section{Results}

\section{Study selection}

The initial search resulted in 3099 titles from all mentioned sources. Following first-stage screening of titles and abstracts, 223 articles qualified for full text examination. Three articles were discarded because full text papers were not available, despite attempts to contact the publishers and authors. After full text analysis of the remaining papers, 102 were excluded and 118 articles met the defined inclusion criteria (Fig. 1-flowchart of study selection). (Supporting material 4-list of excluded studies and reason for exclusion).

An additional 12 articles were identified meeting the inclusion criteria from the reference lists and citations of other included studies. Translation of foreign language manuscripts was completed by colleagues of the authors all of whom also work within the field of dentistry.

The level of agreement between the two reviewers was calculated using kappa statistics for the first and second stage of study selection. The agreement coefficient $(\kappa)$ was 0.88 and 0.83 for the first and second stage, respectively.

\section{Study characteristics}

Of the 118 included studies, 83 were case reports, 33 were case series and 2 were case-control studies. All studies were carried out in a hospital or university environment. A total number of 160 children were reported in the included studies. The number of cases reported in each study varied from 1 to 7 . The age range of included children was 1 to 15 years old. 


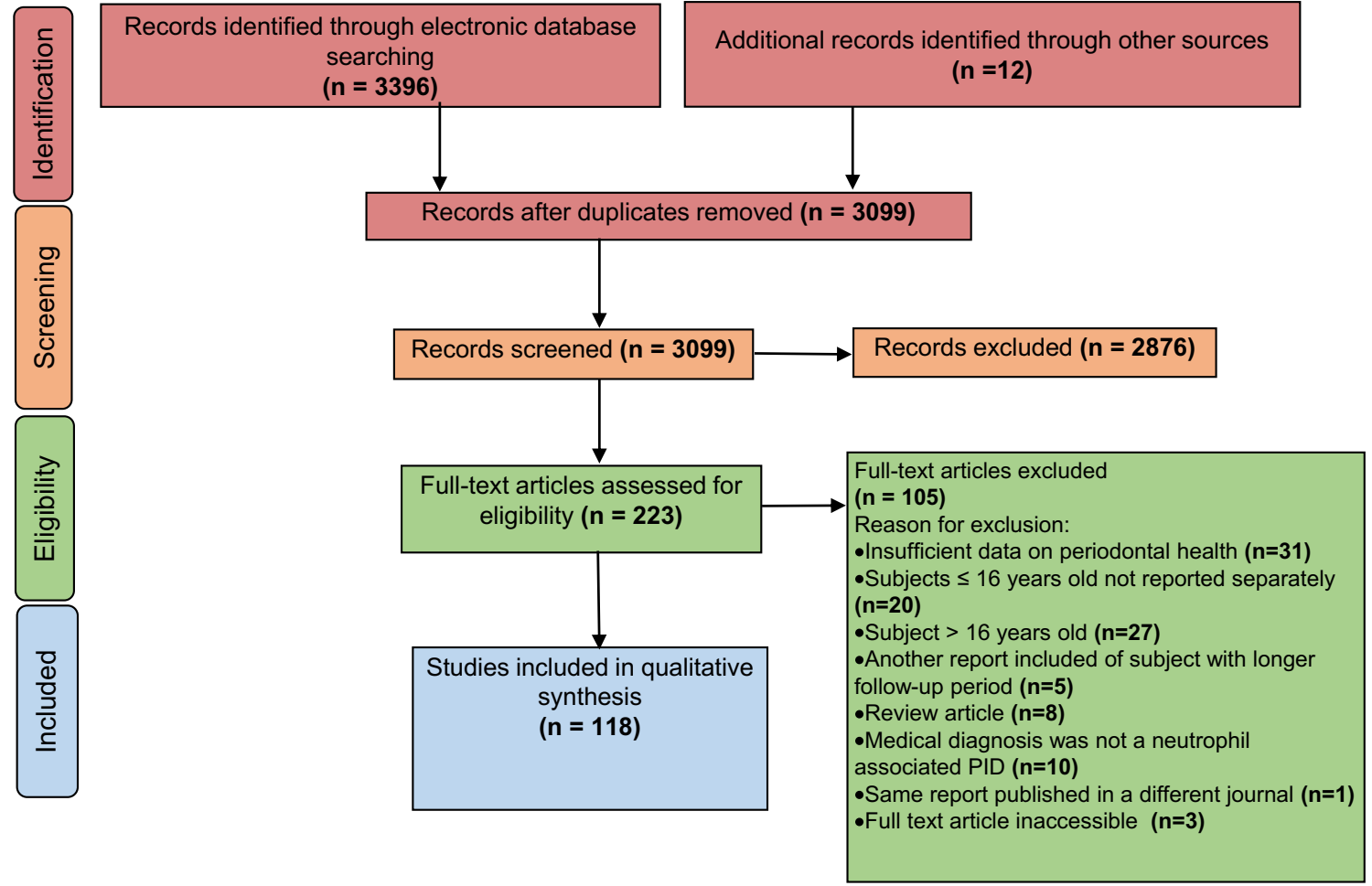

Fig. 1 Study selection flowchart

Eighty-seven (54\%) were female, 72 (45\%) males and in one case sex was not reported. The included PIDs were severe congenital neutropenia $(n=28)$, chronic benign neutropenia $(n=13)$, cyclic neutropenia $(n=22)$, glycogen storage disease $1 \mathrm{~b}(n=5)$, leukocyte adhesion deficiency $(n=11)$, chronic granulomatous disease $(n=1)$, Papillon-Lefèvre syndrome $(n=61)$, Chédiak-Higashi syndrome $(n=8)$, WiskottAldrich syndrome $(n=1)$, Cohen syndrome $(n=3)$ and chronic idiopathic neutropenia $(n=7)$. Most outcomes were measured as narrative descriptions of the child's periodontal condition after periodontal treatment intervention. In some cases, objective measures such as pocket depth reduction, clinical attachment gain, mobility scores, furcation grade, recession grade, plaque and bleeding indices were used. Three studies [12-14] described patient-reported outcomes also. The follow-up period ranged from 6 days to 30 years.

\section{Summary of main findings}

Table 2 reports summary findings for all papers included in this systematic review, arranged by PID diagnosis.

\section{Presenting complaints}

The most common presenting complaints for reported cases was bleeding and sore gums, loose teeth, bad breath and difficulty eating. Presenting complaints were not reported for up to $57 \%$ of cases.

\section{Oral hygiene}

Oral hygiene was reported as poor or suboptimal in up to $57 \%$ of cases. Fair oral hygiene was reported in up to $27 \%$ of included cases. In all other cases, oral hygiene was not reported. Twenty percent of Papillon-Lefèvre syndrome patients presented with halitosis, severe congenital neutropenia, cyclic neutropenia and chronic benign neutropenia patients were reported to have halitosis in 5-10\% of cases.

\section{Gingival inflammation}

All patients with chronic granulomatous disease, ChédiakHigashi syndrome, Cohen syndrome and chronic idiopathic neutropenia presented with severe gingival inflammation. Gingival status was described as 'edematous', 'fiery red', 'hyperplastic' and 'granulomatous'. Three to $18 \%$ of children with severe congenital neutropenia, chronic benign neutropenia, leukocyte adhesion deficiency and Papillon-Lefèvre syndrome were reported to have mild inflammation in the gingival tissues. Five percent of children with cyclic neutropenia and $4 \%$ with severe congenital neutropenia presented with no gingival inflammation. Gingival inflammatory status was not reported in up to $20 \%$ of the included cases. 


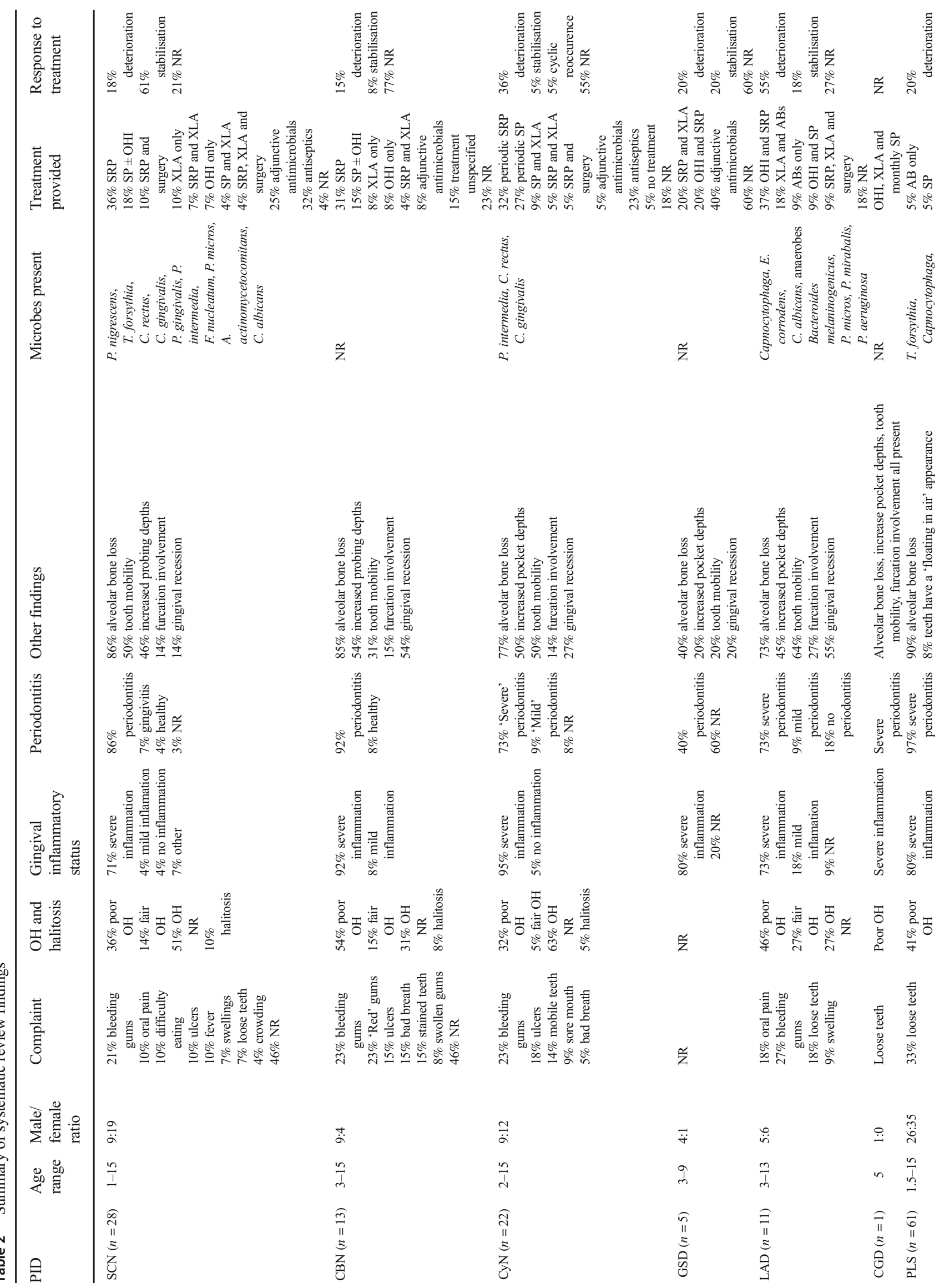




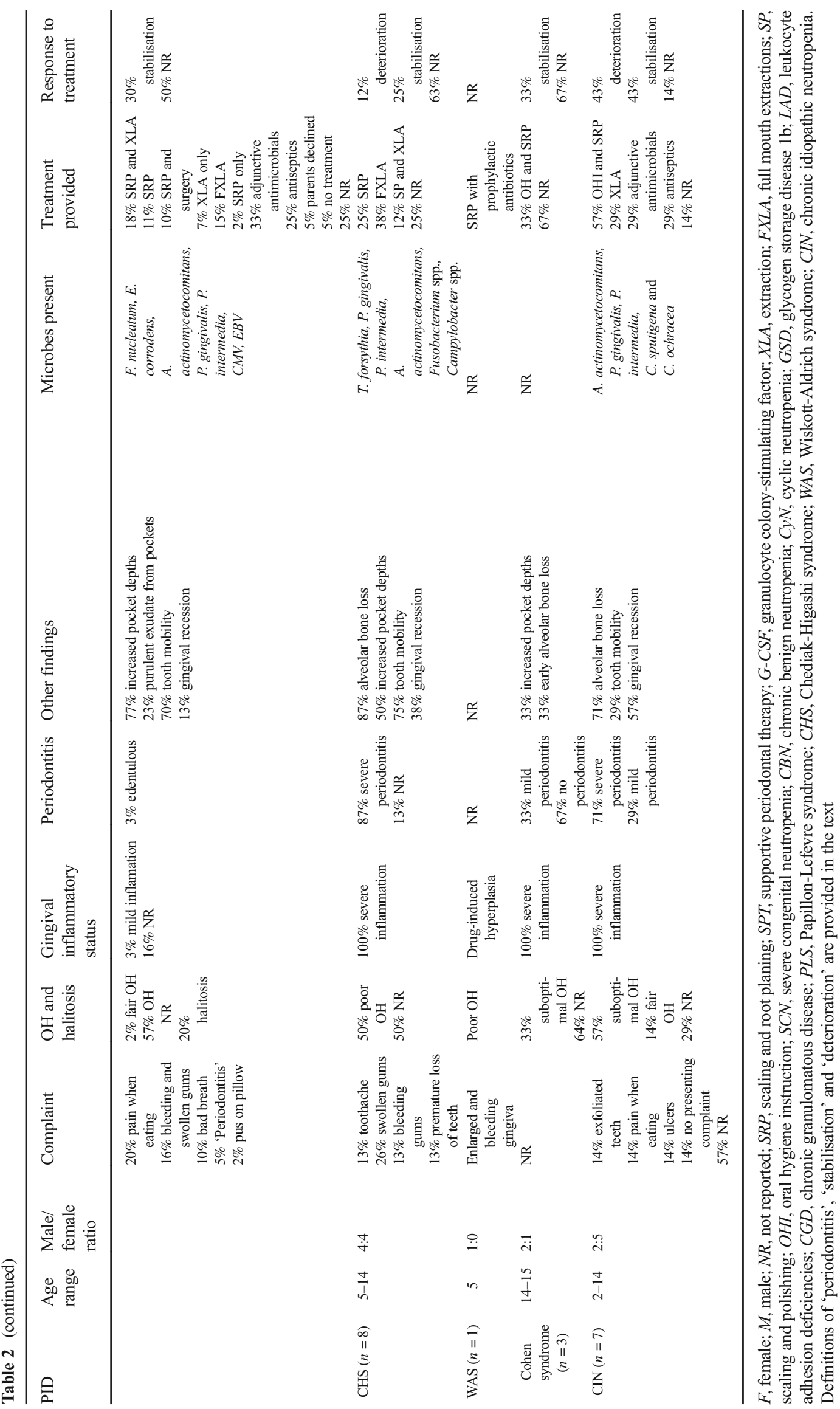




\section{Periodontitis}

In the one case report of chronic granulomatous disease, the child presented with severe periodontitis characterised by alveolar bone loss, increased pocket depths, furcation involvement and tooth mobility. A range of 71-97\% of patients with cyclic neutropenia, leukocyte adhesion deficiency, PapillonLefèvre syndrome, Chédiak-Higashi syndrome and chronic idiopathic neutropenia were described to have a 'severe' level of periodontitis. 'Mild' periodontitis was reported in 9-33\% of cases of severe congenital neutropenia, cyclic neutropenia, leukocyte adhesion deficiency, Cohen syndrome and chronic idiopathic neutropenia. No periodontitis was reported in 4$67 \%$ of patients with severe congenital neutropenia, chronic benign neutropenia, leukocyte adhesion deficiency and Cohen syndrome. The characteristics of periodontal disease in these patients were reported as alveolar bone loss in up to $90 \%$ of cases, increased probing depths in up to $77 \%$ of cases, tooth mobility in up to $75 \%$ of cases, furcation involvement in up to $27 \%$ cases and gingival recession in up to $57 \%$ of cases. Twenty-three percent of patients with Papillon-Lefèvre syndrome also presented with purulent exudate from periodontal pockets and severe bone loss to the apex of teeth in $8 \%$ of cases giving a characteristic 'floating in air' appearance.

\section{Microbiological outcomes}

Some papers reported data of microbes detected in periodontal pockets of PID patients. A variety of microbial techniques were employed, including culture, polymerase chain reaction (PCR) techniques, dark-field microscopy [15] and amplified fragment length polymorphism (AFLP) techniques [16]. The microbes detected in cases of severe congenital neutropenia included Prevotella nigrescens, Tanerella forsythia, Campylobacter rectus, Capnocytophaga gingivalis, Porphyromonas gingivalis, Prevotella intermedia, Fusobacterium nucleatum, Peptostreptococcus micros, Aggregatibacter actinomycetocomitans and Candida albicans. In cyclic neutropenia, $P$. intermedia, $C$. rectus and C. gingivalis were detected. In leukocyte adhesion deficiency, Capnocytophaga spp., Eikenella corrodens, C. albicans, anaerobes Bacteroides melaninogenicus, P. micros, Proteus mirabalis and Pseudomonas aeruginosa were found. Children with Papillon-Lefèvre syndrome presented with T. forsythia, Capnocytophaga spp., F. nucleatum, E. corrodens, A. actinomycetocomitans, P. gingivalis, $P$. intermedia, Cytomegalovirus and Epstein-Barr virus. In Chédiak-Higashi syndrome, T. forsythia, P. gingivalis, P. intermedia, A. actinomycetocomitans, Fusobacterium spp. and Campylobacter spp. were found. In chronic idiopathic neutropenia, the presence of A. actinomycetocomitans, P. gingivalis, $P$. intermedia, Capnocytophaga sputigena and Capnocytophaga ochracea was also reported.

\section{Treatment provided}

Up to $57 \%$ of cases were treated with scaling and root planing (SRP). Seven to $29 \%$ of cases were treated with extraction only. Fifteen percent of Papillon-Lefèvre syndrome patients and $37 \%$ of Chédiak-Higashi syndrome patients had full mouth extractions. Periodontal surgery was performed in $10 \%$ of Papillon-Lefèvre syndrome patients, $9 \%$ of leukocyte adhesion deficiency patients, $5 \%$ of cyclic neutropenia and $14 \%$ of severe congenital neutropenia patients. Adjunctive antimicrobials were used in up to $40 \%$ and adjunctive antiseptics in up to $32 \%$ of cases. Periodontal treatment was not reported in up to $67 \%$ of cases.

\section{Treatment response}

'Stabilisation' of the periodontal condition was noted in $61 \%$ of severe congenital neutropenia cases. In all other conditions, 'stabilisation' was noted in up to $43 \%$ of cases. 'Deterioration' occurred in up to $43 \%$ of cases. Cyclic reoccurrence of periodontal disease was apparent in 5\% of cyclic neutropenia cases. Depending on the PID, periodontal treatment response was not reported in up to $67 \%$ of cases. It was not possible to assess any potential associations between treatment response and oral hygiene levels, since these were rarely reported.

\section{Risk of bias/quality assessment}

A summary of the methodological assessment of included studies is described in supporting material 5,6 and 7. Using the CARE checklist, the quality assessment rating for case reports varied from 9 to 27 . Eight case reports were considered to 'low' quality (score $\leq 10$ ), 54 case reports were considered to be of 'medium' quality (score $>10$ and $\leq 20$ ) and 21 case reports were considered to be of 'high' quality evidence (score $>20$ and $\leq 30$ ). The case series were assessed using the modified Delphi checklist and the range for studies was 1 to 12 . Fourteen case series were considered to 'low' quality (score $\leq 6)$ and 19 case series were considered to be of 'medium' quality (score $>6$ and $\leq 12$ ). No case series were considered to be of 'high' quality evidence (score $>12$ and $\leq 18$ ). The two included case-control studies were assessed using a Newcastle-Ottawa Tool and both studies scored 4 out of a possible 8 , and were therefore considered to be of 'medium' quality.

\section{Discussion}

To our knowledge, this is the first systematic review attempting to determine the clinical and radiographic features of periodontal disease and outcomes of periodontal treatment in children with different types of neutrophil-associated PIDs. 
Acknowledging that it is difficult to summarise such data presented in reports spanning seven decades and with heterogenous reporting and writing styles, we have attempted to collate the data to provide some general findings that have been summarised in Table 2.

In the gingival tissues, neutrophils make up more than $95 \%$ of the total number of leukocytes and form the first line defence against the subgingival biofilm [17]. Therefore, any reduction or dysfunction of neutrophils at the gingival crevice, as seen in severe congenital neutropenia, chronic benign neutropenia, cyclic neutropenia and leukocyte adhesion deficiency, leads to a marked increase in host susceptibility to periodontal disease. A recent study has also reviewed the emerging role of neutrophils in the natural resolution of inflammation, including the secretion of pro-inflammatory mediators such as annexin-A1 and interleukin-10. The same study described the presence of apoptotic neutrophil bodies and their soluble mediators in the host tissues as having the potential to switch the microenvironment from pro-inflammatory to pro-resolution. This phenomenon is termed the 'neutrophil alarm bell' [18].

The defective LYST protein in Chediak-Higashi syndrome disrupts effective neutrophil phagocytosis and the presence of intracellular inclusions inhibits the cellular deformability that allows neutrophil diapedesis. As such, Chediak-Higashi syndrome predisposes patients to a high susceptibility of severe periodontal disease ( $87 \%$ of the cases reported here). The persistence of intracellular Fusobacterium spp. associated with periodontal disease have been isolated in ChediakHigashi syndrome, demonstrating their ability to invade neutrophils and exert their proteolytic activity [19].

Children diagnosed with glycogen storage disease and Cohen syndrome also present with a lower prevalence of periodontal disease (33-40\% of cases reported here). This is also in concordance with previous narrative reviews in this area [6, 20]. Although in our review one case of chronic granulomatous disease was included with severe periodontitis, a large study of 368 children and adult chronic granulomatous disease patients in USA found only $2-3 \%$ presented with gingival inflammation and periodontitis [21]. This study was not included in the current review, as it did not separate findings for adults and children. The reduced prevalence of periodontitis seen in such PIDs could be associated with reduced neutrophil-mediated periodontal tissue destruction [22]. In the case of chronic granulomatous disease, the absence of a 'respiratory burst' from neutrophils, although important in killing of periodontal pathogens, may also protect the periodontal tissues from the 'overspill' of free radicals that occurs during the burst.

All of the patients with periodontitis in leukocyte adhesion deficiency were reported to have severe alveolar bone loss. In leukocyte adhesion deficiency-1, there is often an absence of lymphocyte function-associated antigen (LFA)-1 which is expressed on lymphocyte cell surfaces and is involved in cell signalling, antigen presentation and cytotoxic activity. Interestingly, it has also been shown that in LFA-1 deficient mice develop a spontaneous microbial dysbiosis and alveolar bone loss, although it has not been determined whether this leads to or is a result of local tissue inflammation [23].

Microbes shown to be associated with periodontitis were found in patients with severe congenital neutropenia, cyclic neutropenia, leukocyte adhesion deficiency, Papillon-Lefèvre syndrome, Chédiak-Higashi syndrome and chronic idiopathic neutropenia. These included $P$. gingivalis, $P$. intermedia, T. forsythia, F. nucleatum and A. actinomycetemcomitans [24, 25]. P. gingivalis, a 'key stone' pathogen implicated in periodontitis, has been shown to disrupt the expression of chemokines involved in neutrophil recruitment such as IL-8 and E-selectin. This phenomenon has been termed 'local chemokine paralysis' [26] and may explain in part why children with $P$. gingivalis infection present with severe periodontal disease. A. actinomycetocomitans produces potent leukotoxins that have been shown to be important in the pathogenesis of early onset and aggressive periodontal diseases [27]. F. nucleatum is another virulent periodontopathogen that has shown to induce excessive recruitment of neutrophils [26]. The persistence of redundant and excessive neutrophils can lead to necrosis and subsequent release of their harmful enzymatic contents into the periodontal tissues [28]. Furthermore, in 2 cases of severe congenital neutropenia and one case of cyclic neutropenia, the authors reported improved neutrophil levels after the provision of periodontal therapy or dental extractions [29, 30]. Therefore, it could be hypothesised that disruption and removal of the subgingival biofilm in these cases prevented the periodontal pathogens from exerting their harmful effects on the local microenvironment and allowed the neutrophil count to recover.

The included cases were managed with a wide array of treatment protocols and adjunctive antimicrobial regimes. Many cases also used chlorhexidine antiseptic mouthwash as an adjunct to periodontal therapy [31]. In some patients with cyclic neutropenia and severe congenital neutropenia, the use of granulocyte colony-stimulating factor (G-CSF) to stimulate haematopoiesis of neutrophils appeared to reduce the presence of oral ulceration and gingival inflammation [14, 32-34]. In our systematic review, $20 \%$ of the cases reporting periodontal treatment outcomes for these PIDs were treated with G-CSF and showed periodontal stability, and $10 \%$ showed deterioration. It has also been hypothesised that the periodontal disease pathognomonic in Papillon-Lefèvre syndrome arises from a defective epithelial barrier function in the gingival sulcus and consequential infection with periodontopathogenic bacteria (such as Aggregatibacter actinomycetocomitans and Prevotella intermedia) [35, 36]. Tooth loss in such patients has historically been considered inevitable due to the progressive and uncontrollable nature of the periodontal disease. In 
this review, no more than $30 \%$ of Papillon-Lefèvre syndrome patients were reported to have stabilisation of their periodontal condition at follow-up. In some cases, however, early extraction of deciduous teeth and elimination of periodontopathogenic bacteria (with the use of systemic antimicrobials) prior to eruption of the permanent dentition has been suggested as a management protocol that may reduce the periodontal destruction seen in the permanent teeth [37]. The uses of synthetic retinoids, such as Acitretin, have also been advocated to reduce the chronic gingival inflammation by treating imbalances in collagenolytic activity [38]. Another suggested approach is vital root submergence, where teeth are de-coronated and submerged under the gingival tissues in order to maintain alveolar ridge height and facilitate complete denture retention, support and stability [39]. This suggests that prophylactic removal or submergence of teeth and attempts to eliminate pathogenic bacteria with systemic antimicrobials allow resolution of gingival inflammation. There is evidence to suggest that such treatment approaches allow periodontal health to be maintained for up to 9 years $[40,41]$.

In general, the periodontal treatment outcomes for all PIDS are unpredictable and largely independent of the type of treatment provided. The highest rate of reported periodontal disease 'stabilisation' is seen in only $61 \%$ of severe congenital neutropenia cases. The unsuccessful outcomes of protocols targeted at removal or disruption of the biofilm may suggest that mechanisms other than the loss of neutrophil defence against bacteria could contribute to the development of periodontal disease. Such mechanisms include the persistence of redundant neutrophils in the periodontal tissues, the cytotoxic effects of defective neutrophil lysis [28] and the possible role of neutrophils in the natural resolution of inflammation [18].

This systematic review in particular provides a detailed summary of the published papers reporting periodontal disease in children patients with neutrophil-associated PIDs. Although every attempt was made to access full paper articles of the included studies, this was not possible for 3 articles [42-44]. A large number of studies were included in this systematic review $(n=118)$, but it is important to note that $70 \%$ were case reports and $28 \%$ were case studies, as such representing some of the lowest levels of quality in the hierarchy of evidence [45]. The quality of the included studies varied greatly with case reports scoring between 9 and 27 out of a maximum of 30 and case series ranging from 1 and 12 out of a maximum of 18 . Generally, the older papers presented less relevant clinical data and were less well structured. Many papers failed to report adequately the outcome of treatment and follow-up of the patient. Very few reports appraised different therapeutic options for the patient, and did not provide justification for the chosen treatment. As shown in this systematic review and as described by others [46], many authors did not mention patient-reported outcomes. With medicine becoming ever more patient centred, patient-reported outcomes are more relevant in case reporting and help to inform treatment and the practice of evidence-based healthcare [47].

In such rare conditions as PIDs, there is often a lack of patients to reach statistically significant sample sizes for intervention studies, and so case reports may present the only way to publish information on these patients. However, case reports cannot be used to generalise findings to larger populations because of a lack of cause-effect relationship and of a representative sample. The CARE guidelines [48] were developed in 2013 to provide transparency, accuracy and consistency in the publication of case reports. Despite this, case reports are still susceptible to significant biases, in particular reporting and publication bias where the papers are only published when patients show conditions or outcomes of interest. Therefore, this systematic review does not comment on the relative prevalence of periodontal disease in patients with PIDs, as this can only be determined through comparative case-control studies, of which there are only 2 in this systematic review. There is a definite need for more homogenous studies that focus on comparing PID patients with and without exposure to periodontal disease over a long recall period to allow for the assessment of patient-related outcomes and ultimately tooth loss in such patients. There are also no highquality trials investigating the outcomes of periodontal treatment in this topic area. Publication of such studies would facilitate a meaningful meta-analysis in this systematic review. The lack of randomised controlled trials for the treatment of the dental diseases in children with PIDs should be highlighted. There is also insufficient evidence relating to patientrelated outcomes of periodontal treatment and the psychological and functional consequences of periodontal disease in these children.

The role neutrophil homeostasis in the pathogenesis of periodontal disease is well documented. Through this systematic review, several case reports and case studies and some case-control studies have been identified that document the effect of neutropenia and neutrophil dysfunction on periodontal health in individual children. This review has highlighted that in some of these rare conditions where the neutrophils are absent or dysfunctional, severe periodontal disease often ensues. Affected patients generally complain of oral pain, bleeding and swollen gums, loose teeth, bad breath and difficulty eating. The clinical and radiographic manifestation of the periodontal disease often includes poor oral hygiene, generalised advanced alveolar bone loss, severe gingival inflammation, increased pocket depths, tooth mobility and gingival recession. In some patients (particularly those suffering from neutropenic diseases), the periodontal destruction appears more advanced around the mandibular incisors and in others around molar teeth. Treatment protocols for periodontal disease management have included dental extractions, scaling and root planing, periodic scaling and prophylaxis, adjunctive 
anitmicrobial or antiseptic therapy and less so, surgical interventions. Unfortunately, the current limited evidence suggests that these conventional approaches have limited benefits and the end result often is deterioration of the condition and eventually tooth loss. In Papillon-Lefèvre syndrome, prophylactic extraction of the primary dentition 6 months prior to eruption of the permanent dentition in younger patients tended to experience less periodontal destruction and tooth loss later in life [37]. The use of topical antimicrobial agents was less successful due to lack of patient compliance [37]. In older patients with erupted permanent dentitions, vital root submergence can successfully maintain alveolar ridge height and facilitate complete denture retention, support and stability [39]. The periodontal stability in such patients using these management approaches have been maintained for up to 9 years [37, 40, 41].

Loss of neutrophil protection against periodontal pathogens may not be the only mechanisms that determines the development of periodontal disease in these children. There is increasing evidence of the role of neutrophils in immune signalling pathways and the natural resolution of chronic inflammation. A better understanding of these functions and their role in the pathogenesis of early onset type periodontal disease may help inform novel therapeutic interventions to help relieve the burden on affected children.

The existing literature base with regard to the periodontal health of patients affected by neutrophil-associated PIDs is populated with mostly case reports and case studies of varying quality. The limitations of these case reports and case studies have been discussed previously. There is a need for higher quality observational studies and/or RCTs that compare the relative risk and document the characteristics of periodontal disease in PID patients compared with healthy controls over longer time periods. Generally, PIDs are caused by singled mutations in known genes. These genes maybe provide an insight into the natural mediators of periodontal health and disease. In turn, they may help inform interventional studies aimed at combining gene therapy, stem-cell transplants or G-CSF with periodontal treatment protocols. The difficulty here lies with the rarity of these conditions and the feasibility of recruiting sufficient numbers of patients to make up representative samples.

Acknowledgements The authors would like to gratefully acknowledge all the colleagues who provided translations: Dr. Elena Oleinik, Dr. Attila Horvath, Dr. Krisztián Kövér and Dr. Dániel Palkovics, Dr. Anne Gussgard, Dr. Anna Krajewski, Dr. Kohji Nagata and Prof. Rodrigo Lopez.

This article was made open access with the support of King's College London.

\section{Compliance with ethical standards}

Conflict of interest The authors declare that they have no conflict of interest.
Ethical approval This article does not contain any studies with human participants or animals performed by any of the authors.

Informed consent For this type of study, formal consent is not required.

Open Access This article is distributed under the terms of the Creative Commons Attribution 4.0 International License (http:// creativecommons.org/licenses/by/4.0/), which permits unrestricted use, distribution, and reproduction in any medium, provided you give appropriate credit to the original author(s) and the source, provide a link to the Creative Commons license, and indicate if changes were made.

\section{References}

1. Aghamohammadi A, Moin M, Rezaei N (2010) History of primary immunodeficiency diseases in Iran. Iran J Pediatr 20(1):16-34

2. Fukui N, Amano A, Akiyama S, Daikoku H, Wakisaka S, Morisaki I (2000) Oral findings in DiGeorge syndrome: clinical features and histologic study of primary teeth. Oral Surg Oral Med Oral Pathol Oral Radiol Endod 89(2):208-215. https://doi.org/10.1067/moe. 2000.103884

3. Ku CL, Dupuis-Girod S, Dittrich AM, Bustamante J, Santos OF, Schulze I, Bertrand Y, Couly G, Bodemer C, Bossuyt X, Picard C, Casanova JL (2005) NEMO mutations in 2 unrelated boys with severe infections and conical teeth. Pediatrics 115 (5):e615-e619. doi:https://doi.org/10.1542/peds.2004-1754

4. McAuliffe NJ, Hunter ML, Kau CH, Hunter B, Knox J (2005) The dental management of a patient with hyperimmunoglobulinemia $\mathrm{E}$ syndrome: a case report. Int J Paediatr Dent 15(2):127-130. https:// doi.org/10.1111/j.1365-263X.2005.00596.x

5. Szczawinska-Poplonyk A, Gerreth K, Breborowicz A, BorysewiczLewicka M (2009) Oral manifestations of primary immune deficiencies in children. Oral Surg Oral Med Oral Pathol Oral Radiol Endod 108(3):e9-e20. https://doi.org/10.1016/j.tripleo.2009.03. 049

6. Nicu EA, Loos BG (2016) Polymorphonuclear neutrophils in periodontitis and their possible modulation as a therapeutic approach. Periodontol 2000 71(1):140-163. https://doi.org/10.1111/prd. 12113

7. Hart TC, Atkinson JC (2007) Mendelian forms of periodontitis. Periodontol 2000 45:95-112. https://doi.org/10.1111/j.1600-0757. 2007.00233.x

8. Bullon P, Pascual A, Fernandez-Novoa MC, Borobio MV, Muniain MA, Camacho F (1993) Late onset Papillon-Lefevre syndrome? A chromosomic, neutrophil function and microbiological study. J Clin Periodontol 20(9):662-667

9. Schmidt JC, Walter C, Rischewski JR, Weiger R (2013) Treatment of periodontitis as a manifestation of neutropenia with or without systemic antibiotics: a systematic review. Pediatr Dent 35(2):E54 E63

10. Moher D, Liberati A, Tetzlaff J, Altman DG, Group P (2009) Preferred reporting items for systematic reviews and meta-analyses: the PRISMA statement. J Clin Epidemiol 62(10):1006-1012. https://doi.org/10.1016/j.jclinepi.2009.06.005

11. McGowan JSM, Lefebvre C (2010) An evidence based checklist for the peer review of electronic search strategies (PRESS EBC). Evid Based Libr Inf Pract 5(1):149-154

12. Hasturk H, Tezcan I, Yel L, Ersoy F, Sanal O, Yamalik N, Berker E (1998) A case of chronic severe neutropenia: oral findings and consequences of short-term granulocyte colony-stimulating factor treatment. Aust Dent J 43(1):9-13 
13. Bimstein E, Mcllwain M, Katz J, Jerrell G, Primosch R (2004) Aggressive periodontitis of the primary dentition associated with idiopathic immune deficiency: case report and treatment considerations. J Clin Pediatr Dent 29(1):27-31

14. Quinn J, Shusterman S, Garcia R (1993) Gingival response to GCSF in a patient with congenital agranulocytosis: case report. Pediatr Dent 15(2):123-125

15. van Winkelhoff AJ, Schouten-van Meeteren AY, Baart JA, Vandenbroucke-Grauls CM (2000) Microbiology of destructive periodontal disease in adolescent patients with congenital neutropenia. A report of 3 cases. J Clin Periodontol 27(11):793-798

16. Koeleman JG, Stoof J, Biesmans DJ, Savelkoul PH, Vandenbroucke-Grauls CM (1998) Comparison of amplified ribosomal DNA restriction analysis, random amplified polymorphic DNA analysis, and amplified fragment length polymorphism fingerprinting for identification of Acinetobacter genomic species and typing of Acinetobacter baumannii. J Clin Microbiol 36(9):25222529

17. Delima AJ, Van Dyke TE (2003) Origin and function of the cellular components in gingival crevice fluid. Periodontol 2000 31:55-76

18. Jones HR, Robb CT, Perretti M, Rossi AG (2016) The role of neutrophils in inflammation resolution. Semin Immunol 28(2): 137-145

19. Delcourt-Debruyne EM, Boutigny HR, Hildebrand HF (2000) Features of severe periodontal disease in a teenager with ChediakHigashi syndrome. J Periodontol 71(5):816-824

20. Deas DE, Mackey SA, McDonnell HT (2003) Systemic disease and periodontitis: manifestations of neutrophil dysfunction. Periodontology 2000 32:82-104

21. Winkelstein JA, Marino MC, Johnston RB Jr, Boyle J, Curnutte J, Gallin JI, Malech HL, Holland SM, Ochs H, Quie P, Buckley RH, Foster CB, Chanock SJ, Dickler H (2000) Chronic granulomatous disease. Report on a national registry of 368 patients. Medicine (Baltimore) 79(3):155-169

22. Kantarci A, Oyaizu K, Van Dyke TE (2003) Neutrophil-mediated tissue injury in periodontal disease pathogenesis: findings from localized aggressive periodontitis. J Periodontol 74(1):66-75. https:// doi.org/10.1902/jop.2003.74.1.66

23. Hajishengallis LJ (2011) Microbial manipulation of receptor crosstalk in innate immunity. Nat Rev Immunol 11(3):187-200. https://doi.org/10.1038/nri2918

24. Armitage GC (2010) Comparison of the microbiological features of chronic and aggressive periodontitis. Periodontol 2000(53):70-88. https://doi.org/10.1111/j.1600-0757.2010.00357.x

25. Socransky SS, Haffajee AD, Cugini MA, Smith C, Kent RL Jr (1998) Microbial complexes in subgingival plaque. J Clin Periodontol 25(2):134-144

26. Darveau RP, Belton CM, Reife RA, Lamont RJ (1998) Local chemokine paralysis, a novel pathogenic mechanism for Porphyromonas gingivalis. Infect Immun 66(4):1660-1665

27. Haraszthy VI, Hariharan G, Tinoco EM, Cortelli JR, Lally ET, Davis E, Zambon JJ (2000) Evidence for the role of highly leukotoxic Actinobacillus actinomycetemcomitans in the pathogenesis of localized juvenile and other forms of early-onset periodontitis. J Periodontol 71(6):912-922. https://doi.org/10.1902/jop. 2000.71.6.912
28. Crawford JM, Wilton JM, Richardson P (2000) Neutrophils die in the gingival crevice, periodontal pocket, and oral cavity by necrosis and not apoptosis. J Periodontol 71(7):1121-1129. https://doi.org/ 10.1902/jop.2000.71.7.1121

29. Tozum TF, Berker E, Ersoy F, Tezcan I, Sanal O (2003) The relationship between periodontal status and peripheral levels of neutrophils in two consanguineous siblings with severe congenital neutropenia: case reports. Quintessence international (Berlin, Germany : 1985) 34(3):221-226

30. da Fonseca MA, Fontes F (2000) Early tooth loss due to cyclic neutropenia: long-term follow-up of one patient. Special care in dentistry : official publication of the American Association of Hospital Dentists, the Academy of Dentistry for the Handicapped, and the American Society for Geriatric Dentistry 20(5):187-190

31. Supranoto SC, Slot DE, Addy M, Van der Weijden GA (2015) The effect of chlorhexidine dentifrice or gel versus chlorhexidine mouthwash on plaque, gingivitis, bleeding and tooth discoloration: a systematic review. Int J Dent Hyg 13(2):83-92. https://doi.org/10. 1111/idh. 12078

32. Lu RF, Meng HX (2012) Severe periodontitis in a patient with cyclic neutropenia: a case report of long-term follow-up. The Chinese Journal of Dental Research : the official journal of the Scientific Section of the Chinese Stomatological Association (CSA) 15(2):159-163

33. Nakai Y, Ishihara C, Ogata S, Shimono T (2003) Oral manifestations of cyclic neutropenia in a Japanese child: case report with a 5year follow-up. Pediatr Dent 25(4):383-388

34. Sorin M (1993) Cyclic neutropenia: dental observations, treatment with granulocyte colony-stimulating factor. J Clin Pediatr Dent 17: 183-183

35. Preus HR (1988) Treatment of rapidly destructive periodontitis in Papillon-Lefevre syndrome. Laboratory and clinical observations. J Clin Periodontol 15(10):639-643

36. Meyle J, Gonzales JR (2001) Influences of systemic diseases on periodontitis in children and adolescents. Periodontology 2000 26: 92-112

37. Ullbro C, Brown A, Twetman S (2005) Preventive periodontal regimen in Papillon-Lefevre syndrome. Pediatr Dent 27(3):226-232

38. Lee MR, Wong LCF, Fischer GO (2005) Papillon-Lefevre syndrome treated with acitretin. Australas J Dermatol 46(3):199-201

39. Lu HK, Lin CT, Kwan HW (1987) Treatment of a patient with Papillon-Lefevre syndrome. A case report. J Periodontol 58(11): 789-793. https://doi.org/10.1902/jop.1987.58.11.789

40. Preus H, Gjermo P (1987) Clinical management of prepubertal periodontitis in 2 siblings with Papillon-Lefevre syndrome. J Clin Periodontol 14(3):156-160

41. Preus HR (1988) Treatment of rapidly destructive periodontitis in Papillon-Lefèvre syndrome. J Clin Periodontol 15(10):639-643

42. Baer PN, Iacono VJ (1994) Cyclic neutropenia: report of a case with a 15 -year follow up. Periodontal Clinical Investigations : official publication of the Northeastern Society of Periodontists 16(1): 14-19

43. Gauba M (1960) Periodontosis - a case report of an eight year old child suffering from advanced periodontosis. J All India Dent Assoc 32:131-133

44. Whynman E (1947) A case of oral manifestation of agranulocytosis. Ann Dent 6(4):230 
45. Guyatt GH, Sackett DL, Sinclair JC, Hayward R, Cook DJ, Cook RJ (1995) Users' guides to the medical literature. IX. A method for grading health care recommendations. Evidence-Based Medicine Working Group. JAMA 274(22):1800-1804

46. Rison RA (2013) A guide to writing case reports for the Journal of Medical Case Reports and BioMed Central Research Notes. J Med Case Rep 7 (1):239. doi:https://doi.org/10.1186/1752-1947-7-239

47. Sackett DL (1997) Evidence-based medicine. Semin Perinatol 21(1):3-5

48. Riley DS, Barber MS, Kienle GS, Aronson JK, von SchoenAngerer T, Tugwell P, Kiene H, Helfand M, Altman DG, Sox H,
Werthmann PG, Moher D, Rison RA, Shamseer L, Koch CA, Sun GH, Hanaway P, Sudak NL, Kaszkin-Bettag M, Carpenter JE, Gagnier JJ (2017) CARE guidelines for case reports: explanation and elaboration document. J Clin Epidemiol 89:218-235. https:// doi.org/10.1016/j.jclinepi.2017.04.026

Publisher's note Springer Nature remains neutral with regard to jurisdictional claims in published maps and institutional affiliations. 